\title{
Positive fibroblast growth factor receptor 3 immunoreactivity is associated with low-grade non-invasive urothelial bladder cancer
}

\author{
CÉDRIC POYET $^{1}$, THOMAS HERMANNS ${ }^{1}$, QING ZHONG $^{2}$, EVA DRESCHER ${ }^{1}$, DANIEL EBERLI ${ }^{1}$, \\ MAXIMILIAN BURGER ${ }^{3}$, FERDINAND HOFSTAEDTER $^{4}$, ARNDT HARTMANN $^{5}$, ROBERT STÖHR ${ }^{5}$, \\ ELLEN C. ZWARTHOFF ${ }^{6}$, TULLIO SULSER ${ }^{1}$ and PETER J. WILD ${ }^{2}$ \\ ${ }^{1}$ Department of Urology, ${ }^{2}$ Institute of Surgical Pathology, University Hospital Zurich, University of Zurich, \\ Zurich CH-8091, Switzerland; ${ }^{3}$ Department of Urology, University Hospital Regensburg; ${ }^{4}$ Institute of Pathology, \\ University of Regensburg, Regensburg 93059, Germany; ${ }^{5}$ Institute of Pathology, University of Erlangen, \\ Erlangen 91054, Germany; ${ }^{6}$ Department of Pathology, Erasmus Medical Center, Rotterdam 3015, The Netherlands
}

Received July 6, 2015; Accepted Accepted August 5, 2015

DOI: $10.3892 / 01.2015 .3691$

\begin{abstract}
In addition to conventional clinicopathological parameters, molecular markers are also required in order to predict the course of disease in patients with urothelial bladder cancer (BC). Little is known about fibroblast growth factor receptor 3 (FGFR3) immunoreactivity and the clinical significance it may possess with regard to $\mathrm{BC}$. The present study aimed to investigate the immunoreactivity of FGFR3 in primary urothelial bladder tumours, with regard to clinicopathological features and FGFR3 mutation status. Tissue microarrays were used to immunohistochemically analyse FGFR3 expression in 255 primary, unselected patients with BC. FGFR3 mutations were detected using SNaPshot analysis. Positive FGFR3 immunoreactivity was identified in 113/207 analysable cases $(54.6 \%)$, and was significantly associated with FGFR3 mutation $(\mathrm{P}<0.001)$, low tumour stage $(\mathrm{P}<0.001)$, low histological grade $(\mathrm{P}<0.001)$ and a papillary growth pattern $(\mathrm{P}<0.001)$. Positive FGFR3 immunostaining $(\mathrm{P}=0.002)$ and FGFR3 mutation $(\mathrm{P}=0.002)$ were found to be significantly associated with increased disease-specific survival following univariate analysis, demonstrating a median follow-up period of 75 months. Using multivariate analyses, FGFR3 immunoreactivity was found not to be independent of classical
\end{abstract}

Correspondence to: Dr. Cédric Poyet, Department of Urology, University Hospital Zurich, University of Zurich, 10 Frauenklinikstrasse, Zürich CH-8091, Switzerland

E-mail: cedric.poyet@usz.ch

Abbreviations: BC, bladder cancer; FGFR, fibroblast growth factor receptor; TMA, tissue microarray; RFS, recurrence-free survival; DSS, disease-specific survival

Key words: fibroblast growth factor receptor 3 protein, urothelial cancer, molecular markers, grading, prognosis pathological parameters. Immunohistochemical expression of FGFR3 is an early occurrence during the carcinogenesis of papillary non-invasive BC. The presence of FGFR3 immunoreactivity in non-invasive papillary urothelial carcinomas may be utilised as an indicator of tumours possessing low-grade features and good prognosis.

\section{Introduction}

The majority of patients (75-80\%) exhibiting bladder cancer (BC) typically present with papillary non-invasive (stage pTa) or early invasive (stage pT1) urothelial tumours. The remaining $20-25 \%$ of patients demonstrate more advanced, muscle-invasive tumours ( $\geq \mathrm{pT} 2$ ) (1). A total of $70 \%$ of patients exhibiting stage pTa or pT1 BC will undergo recurrence, and up to $25 \%$ will develop muscle-invasive tumours (2). Therefore, patients with BC must be carefully monitored for signs of disease recurrence or progression. However, to date, there are no established biomarkers in clinical practice that are able to predict the risk of tumour progression.

Based on genetic data, the 2004 World Health Organization (WHO) classification indicates that urinary bladder neoplasia may be subdivided into low- and high-grade lesions (3). There are two genetic subtypes of $\mathrm{BC}$ tumour, which possess marked differences in their levels of genetic instability, and correspond to morphologically distinct entities (4). The first of these subtypes, the genetically stable category, includes low-grade non-invasive papillary tumours. The second genetic subtype, the genetically unstable category, encompasses high-grade and invasive carcinomas, including pTaG3 and pTis, as well as $\geq \mathrm{pT} 1$, respectively (3). Burger et al (5) compared the WHO 1973 and 2004 tumour classification systems, and each system contributed significant information regarding the progression of $\mathrm{BC}$. The inter-observer variability of the WHO 2004 grading system, however, remains an unsolved problem for surgical pathology.

Fibroblast growth factor receptors (FGFRs) control crucial signalling pathways, which are responsible for numerous 
cellular functions, including proliferation and migration (6). FGFRs have been revealed to possess an oncogenic role in numerous types of cancer (7). By contrast, FGFR signalling may also have a suppressive effect on tumours (6). It has been established that BC possesses a link with FGFR mutations, and $\sim 50 \%$ of BCs possess somatic mutations within the FGFR3 coding sequence (8). Mutation of FGFR3 is a common feature of low-grade, non-invasive papillary urothelial $\mathrm{BC}$, occurring in $\sim 75 \%$ of cases (9-12); while occurring at a markedly lower frequency in high-grade invasive BC $(13,14)$, and rarely with adjacent carcinoma in situ $(9,14)$. Patients exhibiting primary BCs accompanied by an activating FGFR3 mutation had significantly improved disease-specific survival (DSS) when compared with patients without FGFR3 mutations $(11,15)$. FGFR3 and TP53 mutations were revealed to be mutually exclusive and may represent two distinct pathways for the development of BC $(11,16,17)$. In these pathways, bladder cancer lesions with activating FGFR3 mutations represent a type of genetically stable, low-grade, papillary tumour. In addition, amalgamation of the analysis of FGFR3 mutations and Ki-67 immunohistochemistry, defined as molecular grading, was revealed to be superior to other parameters for predicting the progression and survival of patients exhibiting BC (11).

FGFR3 mutation status in BC, and the association of BC with the expression of FGFR3 protein, has previously been examined $(18,19)$. The combination of WHO 2004 grading with FGFR3 mutation status facilitated improved risk stratification for patients exhibiting high-grade, non-muscle-invasive urothelial BC (5). However, studies of FGFR3 immunoreactivity and its clinical significance are uncommon $(20,21)$. Immunohistochemical detection of the FGFR3 receptor may provide a simpler, cheaper and faster approach for histopathological practice, compared with the current method of determination of FGFR3 mutation status. Overall, FGFR3 protein has significant potential for use as a diagnostic and prognostic marker, as well as a potential therapeutic target or screening tool (22).

In order to study the prognostic and diagnostic value of FGFR3 protein expression in urothelial BC, a large series of unselected primary urothelial BC tumours were analysed for FGFR3 immunoreactivity and FGFR3 mutations in association with tumour stage, WHO 2004 grade, multifocality, presence of adjacent carcinoma in situ and patient outcome.

\section{Patients and methods}

Bladder cancer tissue microarray (TMA). As previously described (10), a TMA was generated using 255 consecutive, formalin-fixed, paraffin-embedded, primary urothelial BC tissue samples obtained from the Institute of Pathology, University of Regensburg (Regensburg, Germany). Clinical data were obtained from the Central Tumour Registry, Regensburg (Germany), and by telephone interviews when data could not be located. The Institutional Review Board of the University of Regensburg (Regensburg, Germany) approved the analysis of tissues from human subjects. Haematoxylin and eosin-stained slides of all tumour samples were evaluated by a single surgical pathologist (Dr Arndt Hartman). Tumour stages and grades were assigned according to Union for International Cancer Control and WHO 2004 criteria (www.uicc.org/). The growth pattern was determined for all tumours classified as invasive $(\geq \mathrm{pT} 1)$. Papillary growth was defined by the presence of a papillary tumour component $(\geq 20 \%)$, possessing a histological grade identical to that of the invasive tumour. All other tumours were considered to possess a pattern of solid growth. Clinicopathological data are summarised in Table I. Retrospective clinical follow-up data were available regarding the end-points, defined as recurrence-free survival (RFS) and disease-specific survival (DSS), for all patients, and the median follow-up period was 75 months (range, 0-147 months). The median follow-up period for censored patients was 81 months. Recurrence was defined as the presence of cystoscopically visible tumours, and was further confirmed by histological verification. Data regarding progression-free survival (PFS) were not available.

Immunohistochemistry. Immunohistochemical analysis used an avidin-biotin peroxidase method with a diaminobenzidine chromatogen. Following antigen retrieval (using a microwave oven at $250 \mathrm{~W}$ for $30 \mathrm{~min}$ ), immunohistochemistry was performed using an automated NEXES immunostainer (Ventana Medical Systems, Inc., Tucson, AZ, USA) according to the manufacturer's instructions. The following primary antibody was used: anti-FGFR3 [rabbit monoclonal Immunoglobulin G, clone aa 359-372 (E10234); Spring Bioscience, Fremont, CA, USA; dilution 1:50]. FGFR3 immunoreactivity was scored as either negative or positive, irrespective of the staining intensity. Focal (partly positive stained urothelial tissue on the TMA section) or weak FGFR3 (positive but with reduced intensity) immunoreactivity was also considered to be positive.

FGFR3 mutation analysis. FGFR3 mutation analysis was performed using the SNaPshot method as described previously $(12,22)$, using the ABI PRISM SNaPshot Multiplex Kit (Applied Biosystems, Foster City, CA, USA). Three regions of the FGFR3 gene, comprising all FGFR3 mutations identified in BC (23), were simultaneously amplified in a multiplex polymerase chain reaction (PCR). Briefly, the multiplex PCR was performed in a volume of $15 \mu \mathrm{l}$, containing $1 \mathrm{X}$ PCR buffer, $1.5 \mathrm{mmol} / 1 \mathrm{MgCl}_{2}, 0.5 \mathrm{U}$ Taq polymerase (Promega Corporation, Madison, WI, USA), $0.17 \mathrm{mmol} / 1$ deoxynucleotide triphosphates (Roche Diagnostics, Basel, Switzerland), 10 pmol of exon 7 and exon 15 primers, 7.5 pmol of exon 10 primers (Invitrogen, Carlsbad, CA, USA), 5\% glycerol (Fluka, Buchs SG, Switzerland), and 1-250 ng of genomic DNA. Cycling conditions were as follows: $5 \mathrm{~min}$ at $95^{\circ} \mathrm{C}$, 35 cycles at $95^{\circ} \mathrm{C}$ for $45 \mathrm{sec}, 60^{\circ} \mathrm{C}$ for $45 \mathrm{sec}$, and $72^{\circ} \mathrm{C}$ for $45 \mathrm{sec}$, followed by $10 \mathrm{~min}$ at $72^{\circ} \mathrm{C}$. Following removal of excess primers and dNTPs, 8 SNaPshot primers for detection of 9 FGFR3 mutations were annealed to the PCR products and extended using a labelled dideoxynucleotide. These extended primers were analysed using an ABI PRISM 3100 Genetic Analyzer automatic sequencer (Applied Biosystems, Foster City, CA, USA); the label attached to the incorporated nucleotide indicated the presence or absence of mutations. All mutations were verified using a second independent SNaPshot analysis. 
Table I. Patient and tumour characteristics and results of molecular and immunohistochemical analyses.

\begin{tabular}{l} 
Clinicopathological varia \\
\hline Age at diagnosis, years ${ }^{\mathrm{b}}$ \\
$<70$ \\
$\geq 70$
\end{tabular}

Cases, $\mathrm{n}^{\mathrm{a}}(\%)$

Gender

Female

Male

$191(74.9)$

Tumour stage ${ }^{\mathrm{b}}$

PUNLMP

$22(8.6)$

pTa

pT1

pT2

pT3

pT4

124 (48.6)

48 (18.8)

$56(22.0)$

$2(0.8)$

3 (1.2)

Histological grade

1

2

$81(31.8)$

69 (27.1)

105 (41.2)

Histological grade ${ }^{\mathrm{d}}$

$\begin{array}{lr}\text { Low } & 150(58.8) \\ \text { High } & 105(41.2) \\ \text { Adjacent carcinoma in situ } & \\ \text { No } & 222(87.1) \\ \text { Yes } & 33(12.9) \\ \text { Multiplicity } & \\ \text { Solitary } & 53(20.8) \\ \text { Multifocal } & 202(79.2) \\ \text { Growth pattern } & \\ \text { Papillary } & 207(81.5) \\ \text { Solid } & 47(18.5) \\ \text { FGFR3 gene } & \\ \text { Wild-type } & 110(52.9) \\ \text { Mutation } & 98(47.9) \\ \text { FGFR3 immunohistochemistry } & \\ \text { Negative } & 113(54.6) \\ \text { Positive } & \end{array}$

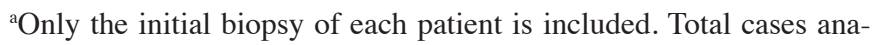
lysed, 255. ' ${ }^{\text {Median, }} 68$ years and range, 34-95 years. ${ }^{\mathrm{c} S t a g i n g}$ and grading according to the WHO 1973 classification system. ${ }^{\text {dStaging }}$ and grading according to the WHO 2004 classification system. FGFR3, fibroblast growth factor receptor 3; PUNLMP, papillary urothelial neoplasia of low malignant potential. Certain cases were not analysed successfully due to insufficient material.

Statistical analysis. Statistical analyses were performed using the survival package in R version 3.0.3 (http://www.r-project. org) and SPSS version 22.0 (IBM SPSS, Armonk, NY, USA). $\mathrm{P}<0.05$ was considered to indicate a statistically significant difference. Associations between the measured parameters were obtained by applying two-sided $\chi^{2}$ and Fisher's exact tests. Non-parametric Kaplan-Meier estimators were used to analyse DSS. Point-wise bands at a confidence level of 0.95 were computed. Differences between survival estimates were evaluated using the Log-Rank test. Cox regression analysis was performed for the variables with regard to DSS. For the analysis of RFS, patients were censored when cystectomy was performed or at the time of their last tumour-free clinical follow-up appointment. For DSS analysis, patients were censored at their final tumour-free clinical follow-up appointment or at their date of mortality unrelated to the tumour.

\section{Results}

Positive FGFR3 immunoreactivity and FGFR3 mutations are associated with positive histopathological characteristics. FGFR3 staining of any intensity was classified as positive, and was observed in 113/207 patients. Positive staining was mainly present in pTa/pT1 tumours (64\%; 89/140). In addition, activating mutations in the FGFR3 gene were detected in 98/208 (47\%) analysable BC tumours, of which 59\% (83/140) were pTa and pT1 tumours.

Table II exhibits the associations between FGFR3 immunohistochemistry and various clinicopathological parameters. A positive FGFR3 staining pattern was largely observed in tumours classified as low-grade and stage pTa. In the low-grade tumour group, $69 \%$ of tumours were FGFR3 positive (Table II) and $74 \%$ carried FGFR3 mutations (data not shown). Fig. 1A demonstrates a typical example of an FGFR3-negative invasive high-grade tumour, while Fig. 1B illustrates an example of an FGFR3 positive non-invasive low-grade tumour. In total, $69 \%$ of pTa lesions were positive for FGFR3, as demonstrated by immunostaining. Fig. 2A and B depict the significant association between tumour stage and grade, and FGFR3 immunoreactivity (both $\mathrm{P}<0.001$ ). Papillary non-invasive (pTa) and low-grade tumours were predominantly positive for FGFR3, as demonstrated by immunohistochemical analysis. There was a significant association between positive FGFR3 immunoreactivity and activating FGFR3 mutations $(\mathrm{P}<0.001$; Fig. 2C).

Positive FGFR3 immunoreactivity was more frequent in tumours that did not posses adjacent carcinoma in situ $(\mathrm{P}=0.062)$. Considering only those tumours with solid growth patterns, which are known to be associated with a worse prognosis (24), positive FGFR3 immunoreactivity was present in a minority of cases $(25 \%$; $\mathrm{P}<0.001)$.

It was concluded that positive FGFR3 staining was associated with low tumour stage and grade, and with a papillary pattern of tumour growth (all $\mathrm{P}<0.001$ ). In a previous study (12), FGFR3 mutation status was observed to be associated with identical clinicopathological parameters such as positive FGFR3 staining in the present study.

FGFR3 is a prognostic biomarker for patients with BC. The end-points for the present study were RFS and DSS. Kaplan-Meier analyses for DSS are exhibited in Fig. 3A and $\mathrm{B}$, and reveal that $\mathrm{BC}$ patients with positive FGFR3 staining had a significantly increased DSS compared with that of patients with negative FGFR3 staining $(\mathrm{P}=0.0018)$. In a subgroup analysis for high-grade tumours, positive FGFR3 
Table II. Comparison of FGFR3 immunoreactivity with clinicopathological and IHC parameters $(\mathrm{n}=207)$.

\begin{tabular}{|c|c|c|c|}
\hline \multirow[b]{2}{*}{ Clinicopathological variable } & \multicolumn{2}{|c|}{ FGFR3 IHC staining pattern } & \multirow[b]{2}{*}{ P-value } \\
\hline & Negative, $\mathrm{n}$ & Positive, $\mathrm{n}$ & \\
\hline Tumour stage $^{\mathrm{a}}$ & & & $<0.001^{\mathrm{c}}$ \\
\hline PUNLMP & 6 & 8 & \\
\hline $\mathrm{pTa}$ & 31 & 68 & \\
\hline pT1 & 20 & 21 & \\
\hline pT2 & 34 & 14 & \\
\hline pT3 & 0 & 2 & \\
\hline pT4 & 3 & 0 & \\
\hline Histological grade ${ }^{a}$ & & & $<0.001^{\mathrm{c}}$ \\
\hline 1 & 23 & 35 & \\
\hline 2 & 13 & 47 & \\
\hline 3 & 58 & 31 & \\
\hline Histological grade ${ }^{b}$ & & & $<0.001^{\mathrm{c}}$ \\
\hline Low & 36 & 82 & \\
\hline High & 58 & 31 & \\
\hline Adjacent carcinoma in situ ${ }^{\mathrm{b}}$ & & & 0.062 \\
\hline No & 77 & 103 & \\
\hline Yes & 17 & 10 & \\
\hline Multiplicity $^{\mathrm{b}}$ & & & 1.000 \\
\hline Solitary & 20 & 25 & \\
\hline Multifocal & 74 & 88 & \\
\hline Growth pattern ${ }^{\mathrm{b}, \mathrm{d}}$ & & & $<0.001^{\mathrm{c}}$ \\
\hline Papillary & 63 & 103 & \\
\hline Solid & 30 & 10 & \\
\hline
\end{tabular}

${ }^{\mathrm{a}} \chi^{2}$-test (two-sided); ${ }^{\mathrm{b}}$ Fisher's exact test (two-sided). ${ }^{\mathrm{c} A}$ statistically significant difference. ${ }^{\mathrm{d}}$ One sample could not be evaluated for papillary or solid tumor growth. PUNLMP, papilllary urothelial neoplasia of low malignant potential; FGFR3, fibroblast growth factor receptor 3; IHC, immunohistochemistry.

A

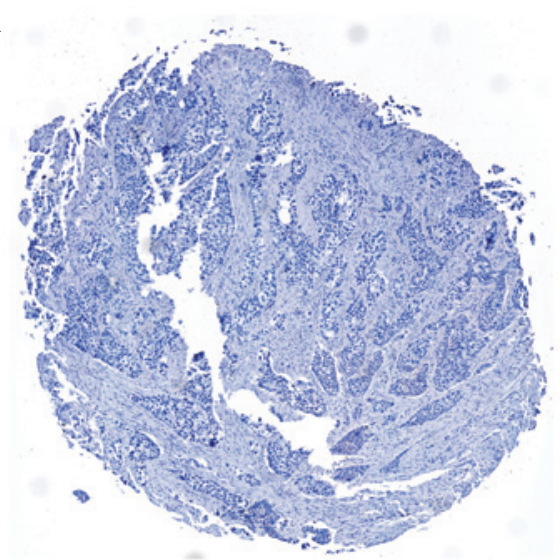

B

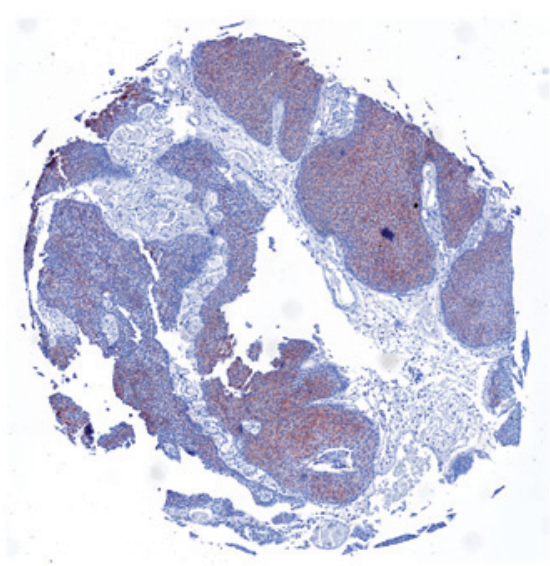

Figure 1. Immunohistochemical staining with FGFR3 antibody. (A) Representative image of negative FGFR3 staining in a high-grade invasive urothelial tumour. (B) Representative imageof positive FGFR3 immunoreactivity in a low-grade non-invasive urothelial bladder cancer specimen. FGFR3, fibroblast growth factor receptor 3 .

staining demonstrated a trend for improved prognosis (Fig. 3B), however this trend was not statistically significant $(\mathrm{P}=0.0991)$.
Fig. 3C summarises the results of the univariate survival analysis for DSS and indicates that positive FGFR3 staining is associated with longer DSS (hazard ratio, 0.35; 95\% 
A

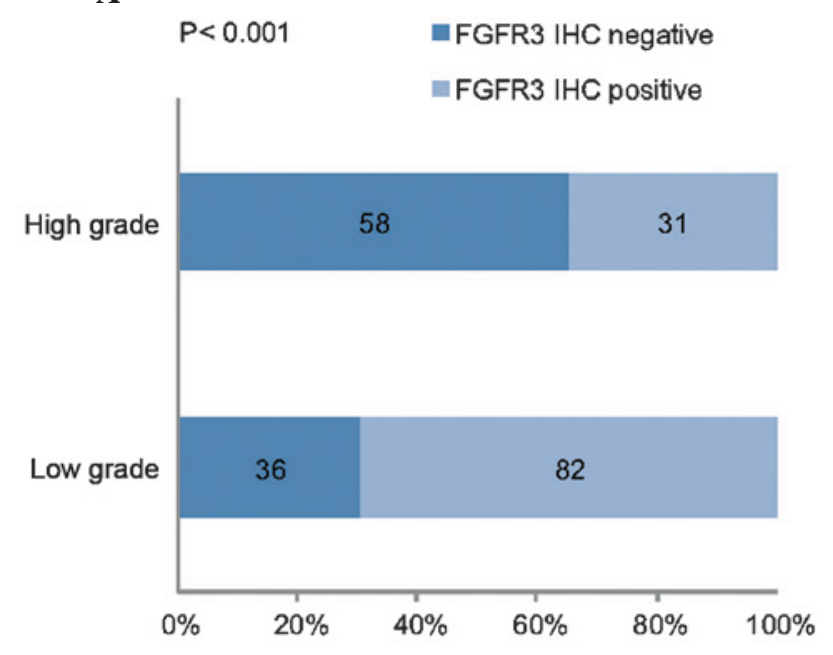

B

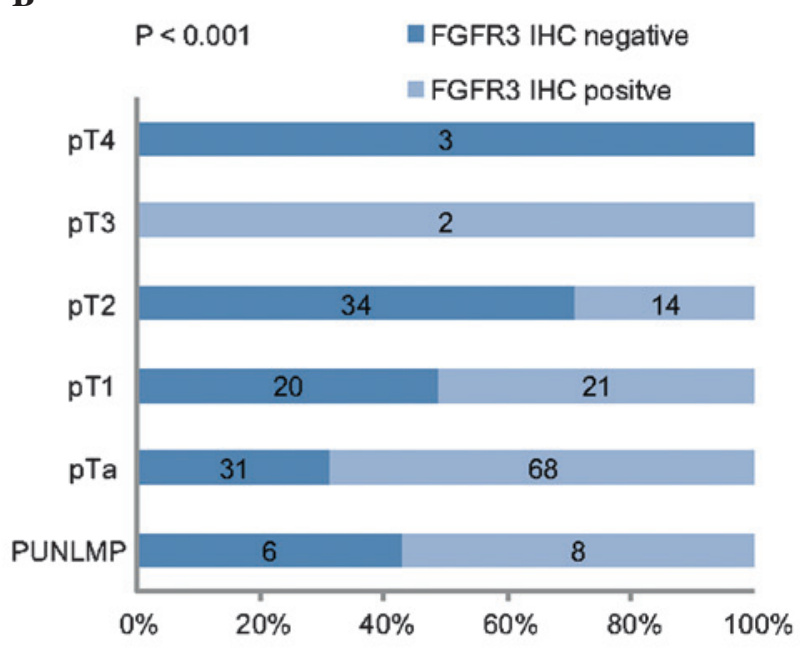

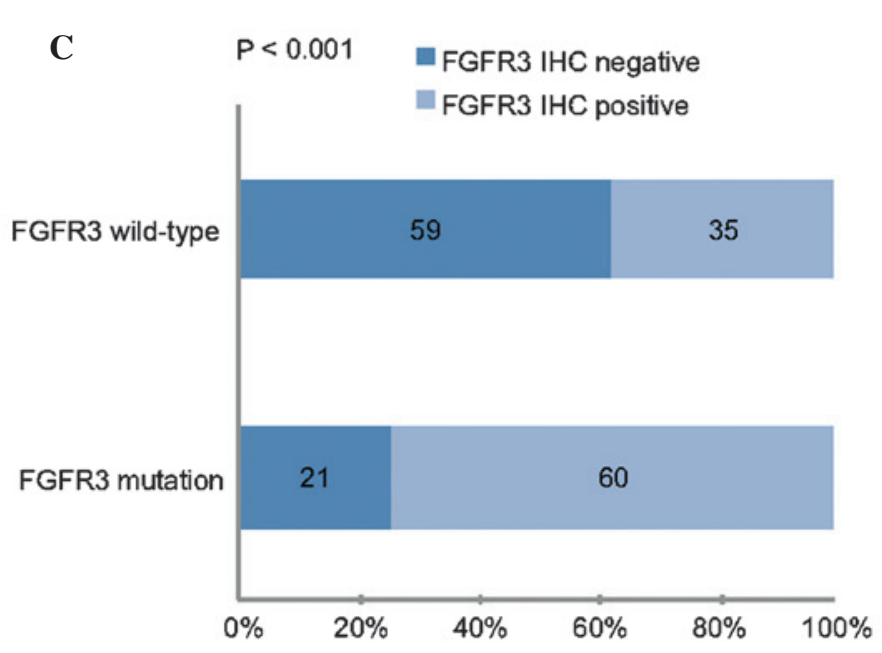

Figure 2. Comparison of FGFR3 staining intensity with (A) grade, (B) stage and (C) FGFR3 mutation of tumours. Frequency represents the percentage of tumours in a particular stage or grade. Dark blue bars represent FGFR3 negative staining and light blue bars represent FGFR3 positive staining. FGFR3, fibroblast growth factor receptor 3; IHC, immunohistochemistry; PUNLMP, papilllary urothelial neoplasia of low malignant potential.

confidence interval, 0.18-0.70; $\mathrm{P}=0.0030)$. Multivariate Cox regression analysis revealed that FGFR3 immunoreactivity was not observed to be a significant parameter associated with DSS (data not shown). None of the investigated parameters demonstrated a correlation with RFS (data not shown).

\section{Discussion}

The present study aimed to investigate FGFR3 immunoreactivity as a potential prognostic biomarker and diagnostic tool in surgical pathology, for patients with BC. The results of the present study revealed that FGFR3 immunoreactivity of any intensity was markedly associated with a low tumour grade and stage, and longer DSS.

In the present study, increased protein expression was identified in $69 \%$ of pTa and $51 \%$ of pT 1 tumours. This finding corresponds with the results of previous studies, which have previously investigated FGFR3 protein expression using immunohistochemistry, which have reported similar percentages of increased FGFR3 protein expression to those observed in the present study $(18,19,23)$. Accordingly, the aforementioned studies identified a significant association between FGFR3 expression and tumour stage and grade.
By contrast, Matsumoto et al (25) identified no association between FGFR3 expression levels and pathological parameters, including grade and stage, potentially due to the low number of cases evaluated leading to a low statistical power $(n=126)$.

Furthermore, the present study demonstrated a correlation between FGFR3 expression and FGFR3 mutation. In the present study, 74\% of tumours with positive FGFR3 expression demonstrated an FGFR3 mutation. This association has additionally been verified using semi-quantitative reverse transcription-PCR, where FGFR3 messenger RNA expression was clearly associated with FGFR3 mutation status (26). Similarly to the results of the present study, Tomlinson et al (18) demonstrated a significant association between FGFR3 expression levels and FGFR3 mutation, as well as tumour grade and stage, respectively.

Previously, FGFR3 mutation status has been demonstrated to be a marker for the prognosis of patients with $\mathrm{BC}(11,27,28)$. In the present study, the correlation between FGFR3 mutations and tumours of low stage and grade was confirmed $(9,11)$. Previous studies have investigated the use of FGFR3 expression as a predictor of prognosis in $\mathrm{BC}$. While a number of authors were able to demonstrate a shorter RFS associated 
A

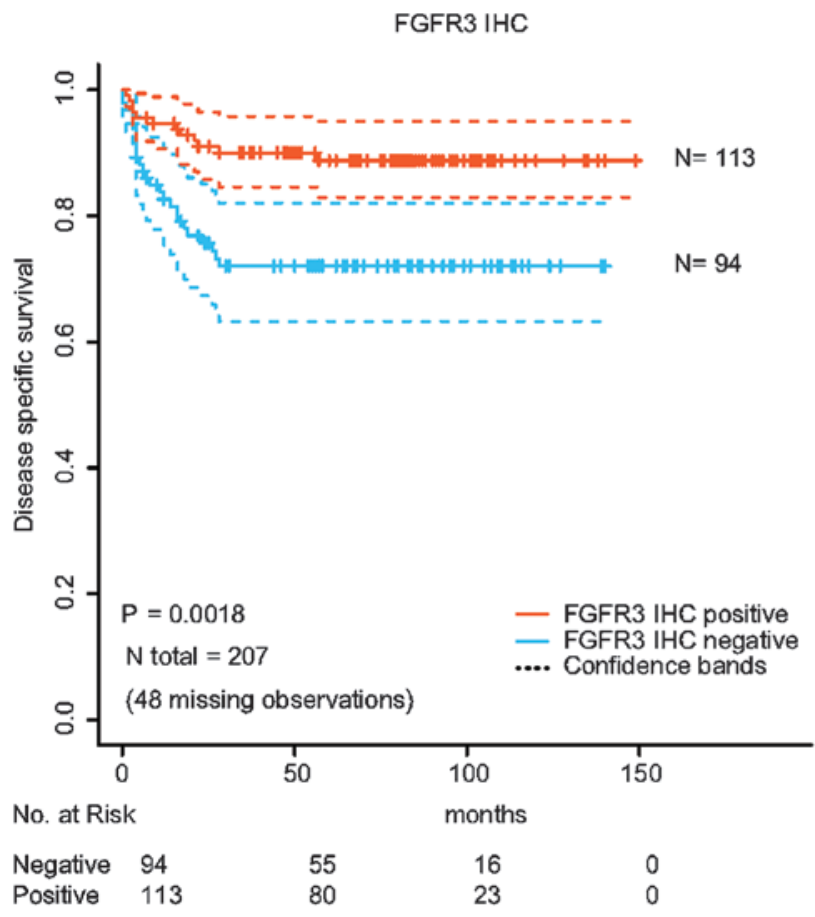

B

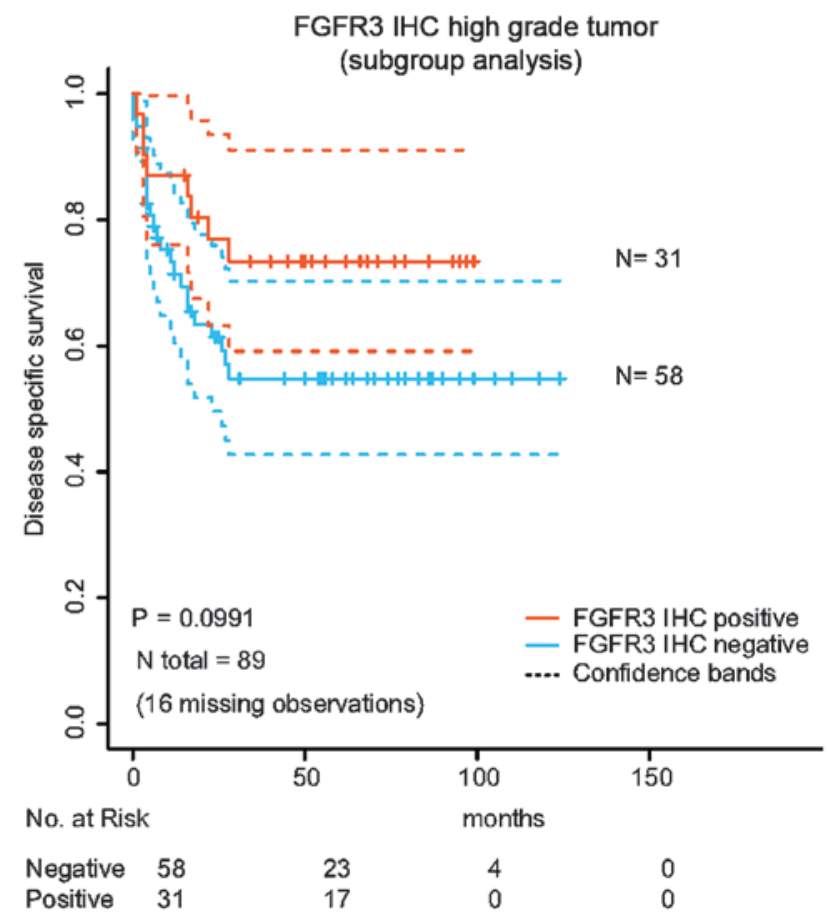

C

Forest plot: univariate Cox regression

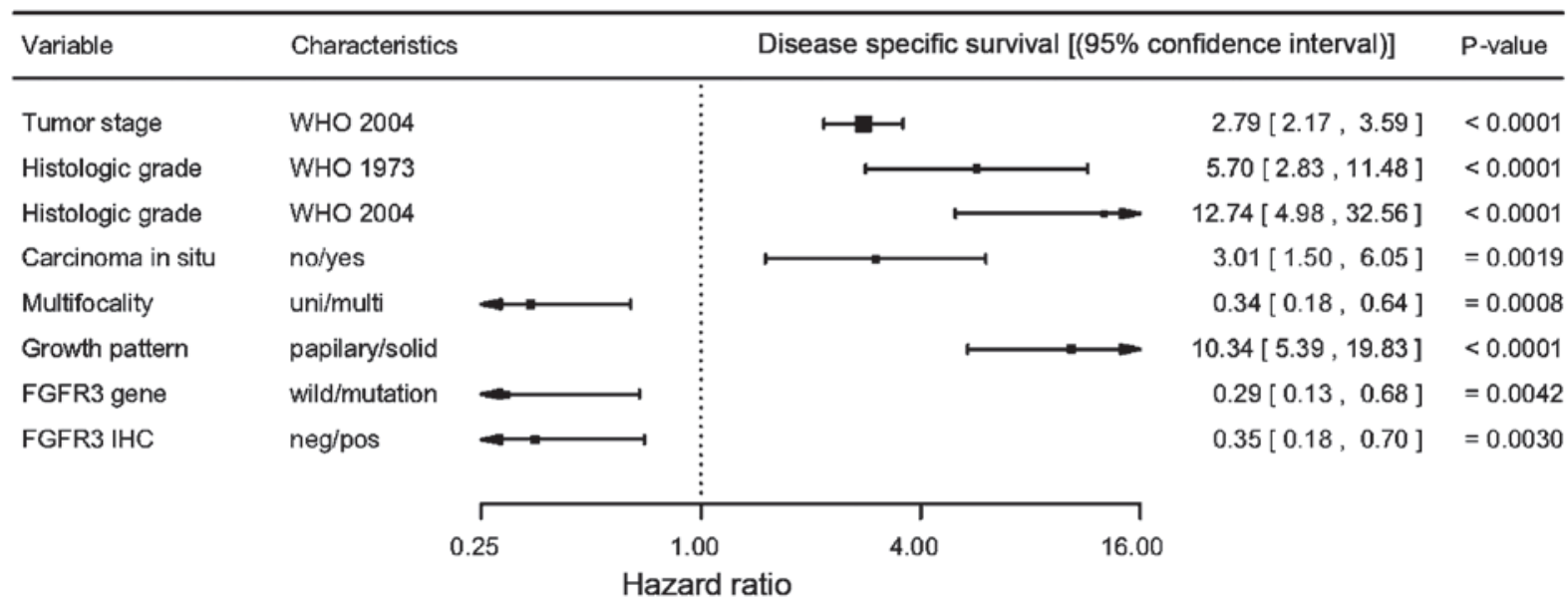

Figure 3. Kaplan-Meier survival plots with point-wise confidence bands (confidence level, 0.95) for disease-specific survival based on FGFR3 staining in (A) all ( $n=207)$ immunohistochemically analysable patients and in (B) a subgroup analysis $(n=89)$ considering only high-grade tumours. Log-Rank tests were performed to test for equality in the survival expectation of each group. $\mathrm{N}$-values represent the number of patients in each group. (C) A forest plot for the univariate Cox regression analysis of factors potentially affecting disease-specific survival of patients with urothelial bladder cancer. The dashed vertical line indicates the no effect point (a hazard ratio of 1.0). Horizontal lines represent $95 \%$ confidence intervals. The midpoint and the area of the box illustrate the mean effect estimate and the weight of each subgroup. FGFR3, fibroblast growth factor 3; IHC, immunohistochemistry; PUNLMP, papilllary urothelial neoplasia of low malignant potential; WHO, world health organisation; uni/multi, solitary/multifocal; neg/pos, negative/positive.

with FGFR3 expression (20,29), Gudjonsson et al (21) did not identify any difference in time to recurrence. In general, the role of molecular markers for predicting $\mathrm{BC}$ recurrence appears to be limited, as reviewed by van Rhijn (30). Even the FGFR3 mutation, which facilitates selective identification of non-muscle-invasive $\mathrm{BC}$ with good prognosis, did not predict recurrence in two studies investigating $>200$ patients, alone or in combination with other molecular markers $(5,11)$. Therefore, it was not noteworthy that the present study was unable to predict recurrence using FGFR3 mutation or expression.
To best of our knowledge, the present study is the first report of an association between FGFR3 protein expression and DSS. The present study demonstrated that in addition to FGFR3 mutation, FGFR3 protein expression was able to predict longer DSS in patients with BC. However, FGFR3 protein expression did not remain an independent predictor of worse DSS following multivariate analysis.

FGFR3 mutation has been proven to be capable of predicting prognosis in several previous studies. Tomlinson et al (18), described the association between 
FGFR3 mutation and protein expression levels, and identified a marked association between FGFR3 protein expression and tumour grade and stage. However, data on progression and DSS were not presented. Bodoor et al (31) studied FGFR3 protein expression and disease course and identified no association between FGFR3 expression and overall survival, although data on DSS was not available. However, two recent studies reported no association between FGFR3 protein expression and prognosis. Guancial et al (32) and Turo et al (33) investigated FGFR3 protein expression in muscle-invasive $\mathrm{BC}$ and identified no association with overall survival. Guancial et al (32) investigated FGFR3 protein expression in 231 primary invasive BCs, while Turo et al (33) studied FGFR3 protein expression in 150 invasive BCs. Guancial et al (32) found FGFR3 mutations in just $2 \%$ of all tumours, whereas Turo et al (33) did not investigate FGFR3 mutation status.

In the present study it was revealed that FGFR3 protein expression was increased in tumours of lower stage and grade. Consequently, FGFR3 protein expression was associated with improved outcomes, as grading and staging are significant, well-known pathological predictors for disease progression in BC.

A significant limitation of the present study was that a major end-point for BC, PFS, was not able to be assessed in this cohort. FGFR3 protein expression was not an independent marker, but was markedly influenced by the pathological parameters, stage and grade for the prediction of disease course. However, FGFR3 protein expression has the potential to serve as an additional molecular marker, alongside tumour grading and staging, in the prediction of prognosis.

FGFR3 mutation analysis using the SNaPshot method has not been implemented in routine practice, due to the high associated costs and complexity. By contrast, FGFR3 immunohistochemistry appears to be more convenient and feasible for use on a routine basis. Analysis of FGFR3 protein expression may provide a tool for use in the assessment of the prognosis of patients exhibiting BC. The present study proposes to use FGFR3 immunoreactivity as an additional diagnostic measure for grading in difficult cases, to better differentiate between low- and high-grade urothelial lesions.

The prognosis of non-muscle-invasive $\mathrm{BC}$ relies on clinicopathological variables to predict outcomes. The present study revealed that the FGFR3 receptor is a significant, but not independent, marker for DSS of BC patients. The present study concluded that FGFR3 protein expression and mutant FGFR3 may provide prognostic information for non-invasive $\mathrm{BC}$, and may aid pathologists with appropriate grading in difficult cases. In addition, loss of FGFR3 expression may identify a subgroup of high-grade tumours with worse prognoses. Further prospective studies evaluating all end-points are required to confirm these data.

\section{Acknowledgements}

The authors would like to thank Rudolf Jung (Institute of Pathology, University of Erlangen, Erlangen, Germany), Martina Storz, Silvia Behnke, Susanne Dettwiler, and Andre Fitsche (All Institute of Surgical Pathology, University Hospital Zurich, University of Zurich, Zurich, Switzerland) for excellent technical assistance and Armin Pauer (Central
Tumor Registry, Regensburg, Germany) for help in obtaining the clinical data.

\section{References}

1. Heney NM: Natural history of superficial bladder cancer. Prognostic features and long-term disease course. Urol Clin North Am 19: 429-433, 1992.

2. Hall MC, Chang SS, Dalbagni G, Pruthi RS, Seigne JD, Skinner EC, Wolf JS Jr and Schellhammer PF: Guideline for the management of nonmuscle invasive bladder cancer (stages Ta, T1, and Tis): 2007 update. J Urol 178: 2314-2330, 2007.

3. Eble J, Sauter G, Epstein JI, Sesterhenn IA (eds): World Health Organization Classification of Tumours. Pathology and Genetics of Tumours of the Urinary System and Male Genital Organs. IARC Press, Lyon, 2004.

4. Wu XR. Urothelial tumorigenesis: A tale of divergent pathways. Nat Rev Cancer 5: 713-725, 2005.

5. Burger M, van der Aa MN, van Oers JM, Brinkmann A, van der Kwast TH, Steyerberg EC, Stoehr R, Kirkels WJ, Denzinger S, Wild PJ, et al: Prediction of progression of non-muscle-invasive bladder cancer by WHO 1973 and 2004 grading and by FGFR3 mutation status: A prospective study. Eur Urol 54: 835-843, 2008 .

6. Turner $\mathrm{N}$ and Grose R: Fibroblast growth factor signalling: From development to cancer. Nat Rev Cancer 10: 116-129, 2010.

7. Dieci MV1, Arnedos M, Andre F and Soria JC: Fibroblast growth factor receptor inhibitors as a cancer treatment: From a biologic rationale to medical perspectives. Cancer Discov 3: 264-279, 2013.

8. Cappellen D, De Oliveira C, Ricol D, de Medina S, Bourdin J, Sastre-Garau X, Chopin D, Thiery JP and Radvanyi F: Frequent activating mutations of FGFR3 in human bladder and cervix carcinomas. Nat Genet 23: 18-20, 1999.

9. Billerey C, Chopin D, Aubriot-Lorton MH, Ricol D, Gil Diez de Medina S, Van Rhijn B, Bralet MP,Lefrere-Belda MA, Lahaye JB, Abbou CC, et al: Frequent FGFR3 mutations in papillary non-invasive bladder (pTa) tumors. Am J Pathol 158: 1955-1959, 2001.

10. van Rhijn BW, Montironi R, Zwarthoff EC, Jöbsis AC and van der Kwast TH: Frequent FGFR3 mutations in urothelial papilloma. J Pathol 198: 245-251, 2002.

11. van Rhijn BW, Vis AN, van der Kwast TH, Kirkels WJ, Radvanyi F, Ooms EC, Chopin DK, Boevé ER, Jöbsis AC and Zwarthoff EC: Molecular grading of urothelial cell carcinoma with fibroblast growth factor receptor 3 and MIB-1 is superior to pathologic grade for the prediction of clinical outcome. J Clin Oncol 21: 1912-1921, 2003.

12. van Oers JM,WildPJ,BurgerM,DenzingerS,StoehrR, RosskopfE, Hofstaedter F, Steyerberg EW, Klinkhammer-Schalke M, Zwarthoff EC, et al: FGFR3 mutations and a normal CK20 staining pattern define low-grade noninvasive urothelial bladder tumours. Eur Urol 52: 760-768, 2007.

13. Hernández S, López-Knowles E, Lloreta J, Kogevinas M, Jaramillo R, Amorós A, Tardón A, García-Closas R, Serra C, Carrato A, et al: FGFR3 and Tp53 mutations in T1G3 transitional bladder carcinomas: Independent distribution and lack of association with prognosis. Clin Cancer Res 11: 5444-5450, 2005.

14. Zieger K, Dyrskjøt L, Wiuf C, Jensen JL, Anderson CL, Jensen KM and Ørntoft TF: Role of activating fibroblast growth factor receptor 3 mutations in the development of bladder tumors. Clin Cancer Res 11: 7709-7719, 2005.

15. van Rhijn BW, van der Kwast TH, Liu L, Fleshner NE, Bostrom PJ, Vis AN, Alkhateeb SS, Bangma CH, Jewett MA, Zwarthoff EC, et al: The FGFR3 mutation is related to favorable pT1 bladder cancer. J Urol 187: 310-314, 2012.

16. van Rhijn BW, van der Kwast TH, Vis AN, Kirkels WJ, Boevé ER, Jöbsis AC and Zwarthoff EC: FGFR3 and P53 characterize alternative genetic pathways in the pathogenesis of urothelial cell carcinoma. Cancer Res 64: 1911-1914, 2004.

17. Bakkar AA, Wallerand H, Radvanyi F, Lahaye JB, Pissard S, Lecerf L, Kouyoumdjian JC, Abbou CC, Pairon JC, Jaurand MC, et al: FGFR3 and TP53 gene mutations define two distinct pathways in urothelial cell carcinoma of the bladder. Cancer Res 63: 8108-8112, 2003.

18. Tomlinson DC, Baldo O, Harnden P and Knowles MA: FGFR3 protein expression and its relationship to mutation status and prognostic variables in bladder cancer. J Pathol 213: 91-98, 2007. 
19. Mhawech-Fauceglia P, Cheney RT, Fischer G, Beck A and Herrmann FR: FGFR3 and p53 protein expressions in patients with pTa and pT1 urothelial bladder cancer. Eur J Surg Oncol 32: 231-237, 2006

20. Maeng YH, Eun SY and Huh JS: Expression of fibroblast growth factor receptor 3 in the recurrence of non-muscle-invasive urothelial carcinoma of the bladder. Korean J Urol 51: 94-100, 2010.

21. Gudjónsson S, Bendahl PO, Chebil G, Höglund M, Lindgren D, Lundberg LM, Lövgren K, Fernö M, Månsson W and Liedberg F: Can tissue microarray-based analysis of protein expression predict recurrence of stage Ta bladder cancer? Scand J Urol Nephrol 45: 270-277, 2011.

22. di Martino E, Tomlinson DC and Knowles MA: A decade of FGF receptor research in bladder cancer: Past, present, and future challenges. Adv Urol 2012: 429213, 2012.

23. Gómez-Román JJ, Saenz P, Molina M, Cuevas González J, Escuredo K, Santa Cruz S, Junquera C, Simón L, Martínez A, Gutiérrez Baños JL, et al: Fibroblast growth factor receptor 3 is overexpressed in urinary tract carcinomas and modulates the neoplastic cell growth. Clin Cancer Res 11(2 Pt 1): 459-465, 2005.

24. Poyet C, Jentsch B, Hermanns T, Schweckendiek D, Seifert HH, Schmidtpeter $\approx$ M, Sulser T, Moch H, Wild PJ, Kristiansen G Expression of histone deacetylases 1,2 and 3 in urothelial bladder cancer. BMC Clin Pathol 14: 10, 2014

25. Matsumoto M, Ohtsuki Y, Ochii K, Seike Y, Iseda N, Sasaki T, Okada Y, Kurabayashi A and Furihata M: Fibroblast growth factor receptor 3 protein expression in urothelial carcinoma of the urinary bladder, exhibiting no association with low-grade and/or non-invasive lesions. Oncol Rep 12: 967-971, 2004.

26. Bernard-Pierrot I, Brams A, Dunois-Lardé C, Caillault A, Diez de Medina SG, Cappellen D, Graff G, Thiery JP, Chopin D, Ricol D and Radranyi F: Oncogenic properties of the mutated forms of fibroblast growth factor receptor $3 \mathrm{~b}$. Carcinogenesis 27 : 740-747, 2006.
27. Lopez-Beltran A, Luque RJ, Alvarez-Kindelan J, Quintero A, Merlo F, Requena MJ and Montironi R: Prognostic factors in survival of patients with stage Ta and $\mathrm{T} 1$ bladder urothelial tumors: The role of G1-S modulators (p53, p21Waf1, p27Kip1, cyclin D1, and cyclin D3), proliferation index, and clinicopathologic parameters. Am J Clin Pathol 122: 444-452, 2004.

28. Hernández S, López-Knowles E, Lloreta J, Kogevinas M, Amorós A, Tardón A, Carrato A, Serra C, Malats N and Real FX: Prospective study of FGFR3 mutations as a prognostic factor in nonmuscle invasive urothelial bladder carcinomas. J Clin Oncol 24: 3664-3671, 2006.

29. Barbisan F, Santinelli A, Mazzucchelli R, Lopez-Beltran A, Cheng L, Scarpelli M, van der Kwast T and Montironi R: Strong immunohistochemical expression of fibroblast growth factor receptor 3 , superficial staining pattern of cytokeratin 20, and low proliferative activity define those papillary urothelial neoplasms of low malignant potential that do not recur. Cancer 112: 636-644, 2008.

30. van Rhijn BW: Combining molecular and pathologic data to prognosticate non-muscle-invasive bladder cancer. Urol Oncol 30: 518-523, 2012.

31. Bodoor K, Ghabkari A, Jaradat Z, Alkhateeb A, Jaradat S, Al-Ghazo MA, Matalka I, Musleh H and Haddad Y: FGFR3 mutational status and protein expression in patients with bladder cancer in a Jordanian population. Cancer Epidemiol 34: 724-732, 2010.

32. Guancial EA, Werner L, Bellmunt J, Bamias A, Choueiri TK, Ross R, Schutz FA, Park RS, O’Brien RJ, Hirsch MS, et al: FGFR3 expression in primary and metastatic urothelial carcinoma of the bladder. Cancer Med 3: 835-844, 2014

33. Turo R, Harnden P, Thygesen H, Fleischmann A, Thalmann GN, Seiler R, Cross WR and Knowles MA: FGFR3 expression in primary invasive bladder cancers and matched lymph node metastases. J Urol 193: 325-330, 2015. 